\title{
Clinical presentation of young people ( $10-24$ years old) with brain tumors: results from the international MOBI-Kids study
}

\author{
Angela Zumel-Marne ${ }^{1,2,3} \cdot$ Michael Kundi $^{4} \cdot$ Gemma Castaño-Vinyals ${ }^{1,2,3,5} \cdot$ Juan Alguacil ${ }^{3,6}$. Eleni Th Petridou ${ }^{7,8}$. \\ Marios K. Georgakis ${ }^{7}$. Maria Morales-Suárez-Varela ${ }^{3,9} \cdot$ Siegal Sadetzki $^{10,11} \cdot$ Sara Piro $^{12} \cdot$ Rajini Nagrani $^{13,14}$. \\ Graziella Filippini $^{15} \cdot$ Hans-Peter Hutter $^{4} \cdot$ Rajesh Dikshit $^{13} \cdot$ Adelheid Woehrer $^{16} \cdot$ Milena Maule $^{17}$.

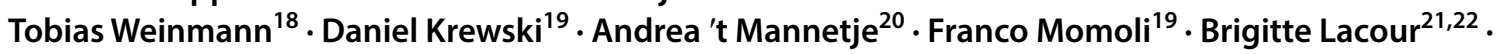 \\ Stefano Mattioli ${ }^{23}$. John J. Spinelli ${ }^{24,25} \cdot$ Paul Ritvo $^{26}$. Thomas Remen ${ }^{21}$. Noriko Kojimahara ${ }^{27}$. Amanda Eng ${ }^{20}$. \\ Angela Thurston $^{20} \cdot$ Hyungryul Lim $^{28} \cdot$ Mina Ha $^{28} \cdot$ Naohito Yamaguchi $^{27}$. Charmaine Mohipp ${ }^{29,30}$. Evdoxia Bouka ${ }^{7}$. \\ Chelsea Eastman $^{1,2,3} \cdot$ Roel Vermeulen $^{31} \cdot$ Hans Kromhout $^{31} \cdot$ Elisabeth Cardis $^{1,2,3}$
}

Received: 8 December 2019 / Accepted: 13 February 2020 / Published online: 3 March 2020

(c) The Author(s) 2020

\begin{abstract}
Introduction We used data from MOBI-Kids, a 14-country international collaborative case-control study of brain tumors (BTs), to study clinical characteristics of the tumors in older children (10 years or older), adolescents and young adults (up to the age of 24).

Methods Information from clinical records was obtained for 899 BT cases, including signs and symptoms, symptom onset, diagnosis date, tumor type and location.

Results Overall, $64 \%$ of all tumors were low-grade, $76 \%$ were neuroepithelial tumors and $62 \%$ gliomas. There were more males than females among neuroepithelial and embryonal tumor cases, but more females with meningeal tumors. The most frequent locations were cerebellum (22\%) and frontal (16\%) lobe. The most frequent symptom was headaches (60\%), overall, as well as for gliomas, embryonal and 'non-neuroepithelial' tumors; it was convulsions/seizures for neuroepithelial tumors other than glioma, and visual signs and symptoms for meningiomas. A cluster analysis showed that headaches and nausea/ vomiting was the only combination of symptoms that exceeded a cutoff of $50 \%$, with a joint occurrence of $67 \%$. Overall, the median time from first symptom to diagnosis was 1.42 months (IQR $0.53-4.80$ ); it exceeded 1 year in $12 \%$ of cases, though no particular symptom was associated with exceptionally long or short delays.

Conclusions This is the largest clinical epidemiology study of BT in young people conducted so far. Many signs and symptoms were identified, dominated by headaches and nausea/vomiting. Diagnosis was generally rapid but in $12 \%$ diagnostic delay exceeded 1 year with none of the symptoms been associated with a distinctly long time until diagnosis.
\end{abstract}

Keywords Brain tumor $\cdot$ Diagnosis $\cdot$ Symptom $\cdot$ Central nervous system tumor $\cdot$ Clinical characteristic

\section{Introduction}

Brain tumors (BT) are one of the most common tumor types in young people. In 2018, the estimated age-standardized annual rate for brain and central nervous system (CNS)

Electronic supplementary material The online version of this article (https://doi.org/10.1007/s11060-020-03437-4) contains supplementary material, which is available to authorized users.

Juan Alguacil

alguacil@dbasp.uhu.es

Extended author information available on the last page of the article tumors in high income areas was 2.5 per 100,000 persons below the age of 25 [1]. Incidence of BT has risen during the last decades and it is unclear whether this is due solely to improved diagnostic practices or to a real increase in disease frequency $[2,3]$.

Tumor histology and location vary with age. In adults, the most frequent BT subtypes are gliomas located in the supratentorial region-and specifically in the temporal and parietal lobes-while in children, the most frequent are low grade gliomas [4] and embryonal tumors located primarily in the posterior fossa (cerebellum and brainstem) [5]. 
Symptoms preceding BT diagnosis are non-specific, and are often initially attributed to other diseases. Advanced knowledge about common symptoms of BT in the pediatric, adolescent and young adult populations could raise awareness among the medical community [6,7].

The primary objective of this paper is to describe the clinical characteristics of BT in young people using data from 899 cases recruited in the international MOBI-Kids case-control study, focusing on morphology, topography, signs and symptoms and time to diagnosis. We also aim to study whether certain signs and symptoms are associated with specific tumor morphologies and locations, or with variables such as gender or age. We report results for older children (10 years or older), adolescents and young adults (up to the age of 24) (a group referred to as "young people" in this paper) to provide information for those studying childhood as well as young adult oncology.

\section{Material and methods}

MOBI-Kids is a multinational case-control study set-up to estimate risk of BTs in relation to electromagnetic fieldsmainly Extremely Low Frequency and radiofrequency (RF) fields-exposure from use of mobile communication device. Cases were patients with a first primary benign or malignant BT, diagnosed between the ages of 10 and 24 years, during a 3-4 study period between 2010-2016 (depending on country), and residing in one of the study regions of the participating countries (Australia, Austria, Canada, France, Germany, Greece, India, Israel, Italy, New Zealand, Spain, Netherlands, Japan and Korea). Eligible tumors were those originating in areas of the brain that absorb the highest RF energy from mobile phones held by the ear, thus excluding midline tumors (those close to the skull base, mostly pituitary and pineal tumors). Tumors known to be associated with a genetic syndrome were also excluded. Ethics committee approvals for the study were obtained from all national and regional review boards. An informed consent was obtained from all participants. The detailed protocol of MOBI-Kids was previously published [8].

A clinical questionnaire was completed by interviewers trained for this study with the help of neurosurgeons, neuroradiologists and/or pathologists, based on available clinical records including imaging, histopathology, surgery, and on clinical reports. Quality and precision of reports about clinical presentation especially concerning timing of signs and symptoms varied but were typically more precise the closer to diagnosis they occurred. There were some differences among countries concerning participation of neurosurgeons or pathologists in data extraction. Examination of precision and completeness revealed no systematic differences with respect to their participation. In cases where ambiguities could not be solved, the team reviewed the clinical records a second time. In this article we explore the information from the clinical questionnaire concerning the clinical characteristics of the tumors in MOBI-Kids.

Details about data collected, coding and statistical methods are provided in the Supplemental Materials.

\section{Results}

We collected clinical information on 899 cases. Eight did not authorize access to their clinical records and hence the only information available is tumor morphology and topography. Symptoms information was available for 722 cases (two participating countries did not collect information about these) (Fig. 1). Overall, $42 \%$ of cases were aged $10-14$ years at diagnosis, 32\% 15-19 years and 26\% 20-24 years (Table 1), with a higher proportion of males $(57 \%)$ than females.

\section{Tumor characteristics}

The majority of cases had neuroepithelial tumors (76\%) (Table 1). Gliomas represented $62 \%$ of all tumors; other neuroepithelial tumors were a variety of different morphologies, such as ganglioglioma NOS $(n=55)$, dysembryoplastic neuroepithelial tumors $(n=23)$, central neurocytomas $(n=18)$, among other rare tumors. Among non-neuroepithelial tumors, embryonal tumors were the most frequent (14\% of all tumors), followed by meningioma (5\%). Cases with rare morphologies ( $\leq 30$ cases) are described in Supplementary Tables A13-A14.

The distribution of neuroepithelial tumors was similar across age-groups and sex overall (Table 2). Embryonal tumors were more frequent among males and a significant decreasing trend was seen with increasing age. In contrast, meningiomas and other non-neuroepithelial tumors were slightly more frequent among females, with frequency increasing with age.

Regarding topography (Table 2 and Supplementary Figure A1), the most frequent locations were cerebellum, frontal and temporal lobes. The distribution of cases by gender was generally similar by topography. While the proportions of brainstem and cerebellar tumors declined with increasing age, those of tumors of the frontal lobe and cerebral meninges tumors increased with age (Table 2). Gliomas were most frequently located in the frontal lobe $(20 \%)$ and cerebellum $(17 \%)$, while other neuroepithelial tumors arose mainly in the temporal (32\%) and frontal (16\%) lobes and cerebral ventricles (17\%) (Supplementary Table A3). Embryonal tumors occurred mainly in the cerebellum (67\%), while the majority of other non-neuroepithelial tumors, except meningioma, were located in cranial nerves (36\%). 
Fig. 1 Flowchart for collection of information

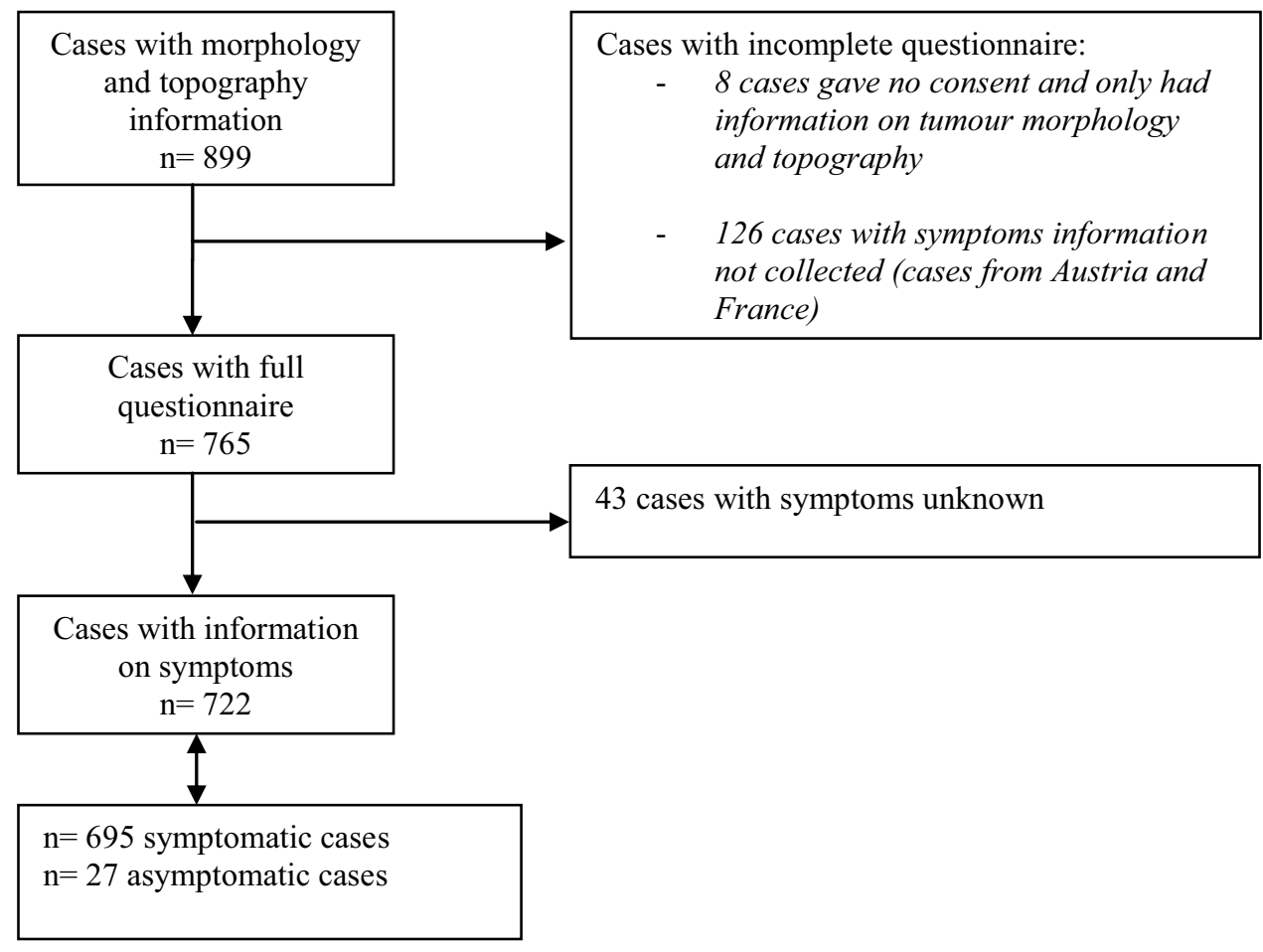

The majority of tumors were low-grade (WHO grades I/II): $69 \%$ of neuroepithelial tumors (gliomas: $64 \%$, other neuroepithelial: $96 \%$ ), 98\% of meningiomas and $100 \%$ of other non-neuroepithelial tumors. All embryonal tumors were high-grade (Supplementary Table A4). For cerebellum, brainstem, and cerebral meninges the frequency of low- and high-grade tumors were similar.

\section{Analysis of symptoms}

Among those with information on symptoms (722 cases), 27 cases were asymptomatic, 165 cases reported 1 symptom, 177 cases 2, 153 cases 3, and 200 more than 3 symptoms (up to 10) before diagnosis. Overall, among symptomatic cases, a median of 3 symptoms was observed (Table 1); the median was lower (2) in cases 15-24 years old, than in younger cases $(\mathrm{p}<0.01)$ (Table 1$)$. Females reported more symptoms $($ median $=3)$ than males $($ median $=2)$.

Statistically significant differences in number of symptoms by morphology were observed. Cases with embryonal tumors had more symptoms (median $=4)$, than cases with other morphologies (median $=2-3$ ). Tumors in the frontal, temporal, occipital lobes, cerebral meninges, cranial nerves and brain NOS had fewer symptoms (median =2) than those located in the brainstem, cerebellum, cerebral ventricles and overlapping lesion of the brain (median $=3$ ), or parietal lobe $($ median $=2.5)$. The number of symptoms tended to be higher in higher grade tumors (Table 1).
For those with at least one symptom, the most frequently reported were headaches $(n=436 ; 60 \%$ of cases with symptom information), focal neurological signs and symptoms $(\mathrm{n}=287 ; 40 \%)$, nausea/vomiting $(\mathrm{n}=277 ; 38 \%)$ and visual signs and symptoms $(n=217 ; 30 \%)$ (Table 3$)$.

Distributions of cases by detailed symptoms and morphology are in Supplementary Table A5. The majority of glioma cases reported headaches (64\% in high-grade, 59\% in low-grade tumors), whereas the most frequent symptoms reported for other neuroepithelial tumors were convulsions/ seizures (53\%) and headaches (46\%). Among non-neuroepithelial tumors, headaches were also the most frequent, except for meningiomas, with visual signs and symptoms (49\%).

Distribution of cases by symptoms and topography are shown in Table 3 and, in details, in Supplementary Table A6 and Figure A2. The frequency of cognitive, memory and behavioral changes did not differ by topography. Headache was reported in $58-82 \%$ of tumors of the brainstem, cerebellum, cerebral ventricles, overlapping lesion of the brain and for brain NOS; the proportion was lower in other anatomical regions of the brain. Nausea and vomiting was reported by $63 \%$ of patients with a tumor in the cerebellum, a higher proportion than in other locations. Focal neurological signs and symptoms were mentioned for $65 \%$ of patients with a tumor in the brainstem. Convulsions/seizures were reported for $43-61 \%$ of tumors in the frontal, temporal, parietal and occipital lobes but in $5 \%$ or less of tumors of the brainstem, cerebellum, cranial 
Table 1 Number of cases and cases with reported symptoms, median number of symptoms by age, sex, morphology, topography and grade of tumor

\begin{tabular}{|c|c|c|c|c|c|}
\hline & \multirow{2}{*}{$\begin{array}{l}\text { Cases }(\mathrm{n}=899)^{\mathrm{a}} \\
\mathrm{n}(\%)\end{array}$} & \multirow{2}{*}{$\begin{array}{l}\text { Cases with symptom infor- } \\
\text { mation }(\mathrm{n}=722)^{\mathrm{b}} \\
\mathrm{n}(\%)\end{array}$} & \multirow{2}{*}{$\begin{array}{l}\text { Symptomatic cases } \\
(\mathrm{n}=695)^{\mathrm{c}} \\
\mathrm{n}(\%)\end{array}$} & \multicolumn{2}{|c|}{$\begin{array}{l}\text { Number of symptoms reported } \\
(\mathrm{n}=695)\end{array}$} \\
\hline & & & & $\overline{\text { Median (IQR) }}$ & p-value ${ }^{\mathrm{d}}$ \\
\hline Age & & & & & $<0.01$ \\
\hline $10-14$ & $375(42)$ & $301(42)$ & $292(97)$ & $3(2-4)$ & \\
\hline $15-19$ & $292(32)$ & $230(32)$ & $221(96)$ & $2(2-3)$ & \\
\hline $20-24$ & $232(26)$ & $191(26)$ & $182(95)$ & $2(1-3)$ & \\
\hline Sex & & & & & 0.03 \\
\hline Male & $512(57)$ & $397(55)$ & $377(95)$ & $2(2-4)$ & \\
\hline Female & $387(43)$ & $325(45)$ & $318(98)$ & $3(2-4)$ & \\
\hline Morphology & & & & & $<0.01$ \\
\hline Gliomas & $556(62)$ & $459(64)$ & $443(97)$ & $2(2-4)$ & \\
\hline Other neuroepithelial & $120(14)$ & $92(13)$ & $90(98)$ & $2(1-3)$ & \\
\hline Embryonal & $129(14)$ & $102(14)$ & $98(96)$ & $4(2-5)$ & \\
\hline Meningiomas & $47(5)$ & $35(5)$ & $32(91)$ & $2(1-3)$ & \\
\hline Other non-neuroepithelial & $47(5)$ & $34(4)$ & $32(94)$ & $3(2-4)$ & \\
\hline Topography & & & & & $<0.01$ \\
\hline Brain stem & $91(10)$ & $68(9)$ & $65(96)$ & $3(2-5)$ & \\
\hline Cerebellum & $201(22)$ & $163(23)$ & $157(96)$ & $3(2-4)$ & \\
\hline Frontal lobe & $143(16)$ & $113(16)$ & $106(94)$ & $2(1-3)$ & \\
\hline Temporal lobe & $103(11)$ & $88(12)$ & 87 (99) & $2(1-3)$ & \\
\hline Parietal lobe & $52(6)$ & $37(5)$ & $36(97)$ & $2.5(2-3)$ & \\
\hline Occipital lobe & $17(2)$ & $16(2)$ & $15(94)$ & $2(1-3)$ & \\
\hline Cerebral ventricles & $88(10)$ & $74(10)$ & $73(99)$ & $3(2-4)$ & \\
\hline Cerebral meninges & $31(4)$ & $24(3)$ & $23(96)$ & $2(1-3)$ & \\
\hline Cranial nerves & $30(3)$ & $21(3)$ & $20(95)$ & $2(1-4)$ & \\
\hline Overlapping lesion of the brain & $83(9)$ & $76(11)$ & $73(96)$ & $3(1-5)$ & \\
\hline Other parts of the brain ${ }^{\mathrm{d}}$ & $60(7)$ & $42(6)$ & $40(95)$ & $2(2-3)$ & \\
\hline Grade of tumor (WHO) & & & & & $<0.01$ \\
\hline I & $386(43)$ & $308(42)$ & $295(96)$ & $2(1-4)$ & \\
\hline II & $177(20)$ & $144(20)$ & $137(95)$ & $2(1-3)$ & \\
\hline III & $142(15)$ & $113(16)$ & $111(98)$ & $3(2-4)$ & \\
\hline IV & $194(22)$ & $157(22)$ & $152(97)$ & $3(2-4)$ & \\
\hline Overall & 899 & 722 & 695 & $3(2-4)$ & \\
\hline
\end{tabular}

${ }^{a}$ Percentage calculated based on the total number of 899 cases; ${ }^{b}$ percentage calculated based on the total number of 722 cases with information on symptoms; ' percentage calculated based on row category number of cases with symptom information; ${ }^{\mathrm{d} K r u s k a l-W a l l i s ~ t e s t ~ c o m p a r i n g ~ n u m-~}$

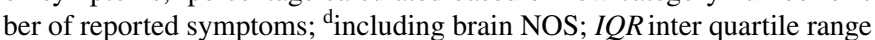

nerves and brain NOS. Dizziness affected mainly cases with brainstem (34\%) or cerebellar (29\%) tumors.

Distributions of symptoms by grade are shown in Table 4 . The proportion of cases with headaches, nausea and vomiting and dizziness varied by grade, being highest for grade IV tumors (headaches: $75 \%$; nausea and vomiting: $61 \%$; dizziness: $27 \%$ ). In contrast, the proportion of cases with convulsions/seizures was lowest in high-grade tumors (12\%). Other symptoms showed little differences by grade. Most asymptomatic tumors (48\%) were grade I and of small size $($ mean $=2.50 \mathrm{~cm})$.
Laterality of symptoms was reported for only $17 \%$ of cases with lateralized symptoms (Supplementary Table A7). Little difference was seen for these symptoms, based on small numbers of cases, except for altered sensitivity, more frequent on the side contralateral to the tumor.

In cluster analyses of the main symptom categories (Supplementary Figures A3-A5), headaches and nausea/vomiting were the only symptoms which met the 50\% statistical cutoff, occurring together for the majority of topographies and morphologies (Dice dissimilarity coefficient, $\mathrm{DDC}=0.33$, equivalent to $67 \%$ joint occurrences). Other, non- significant 


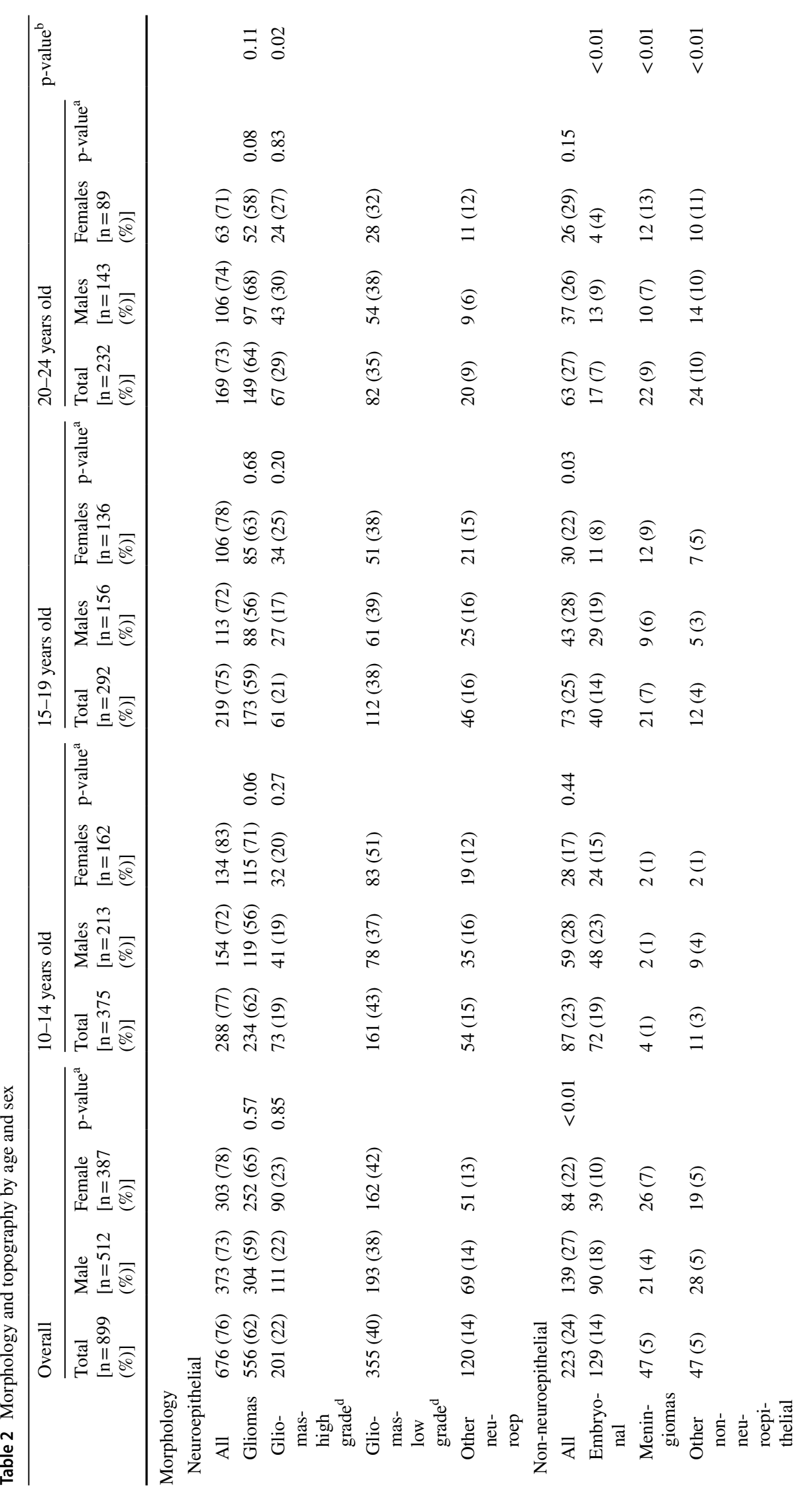




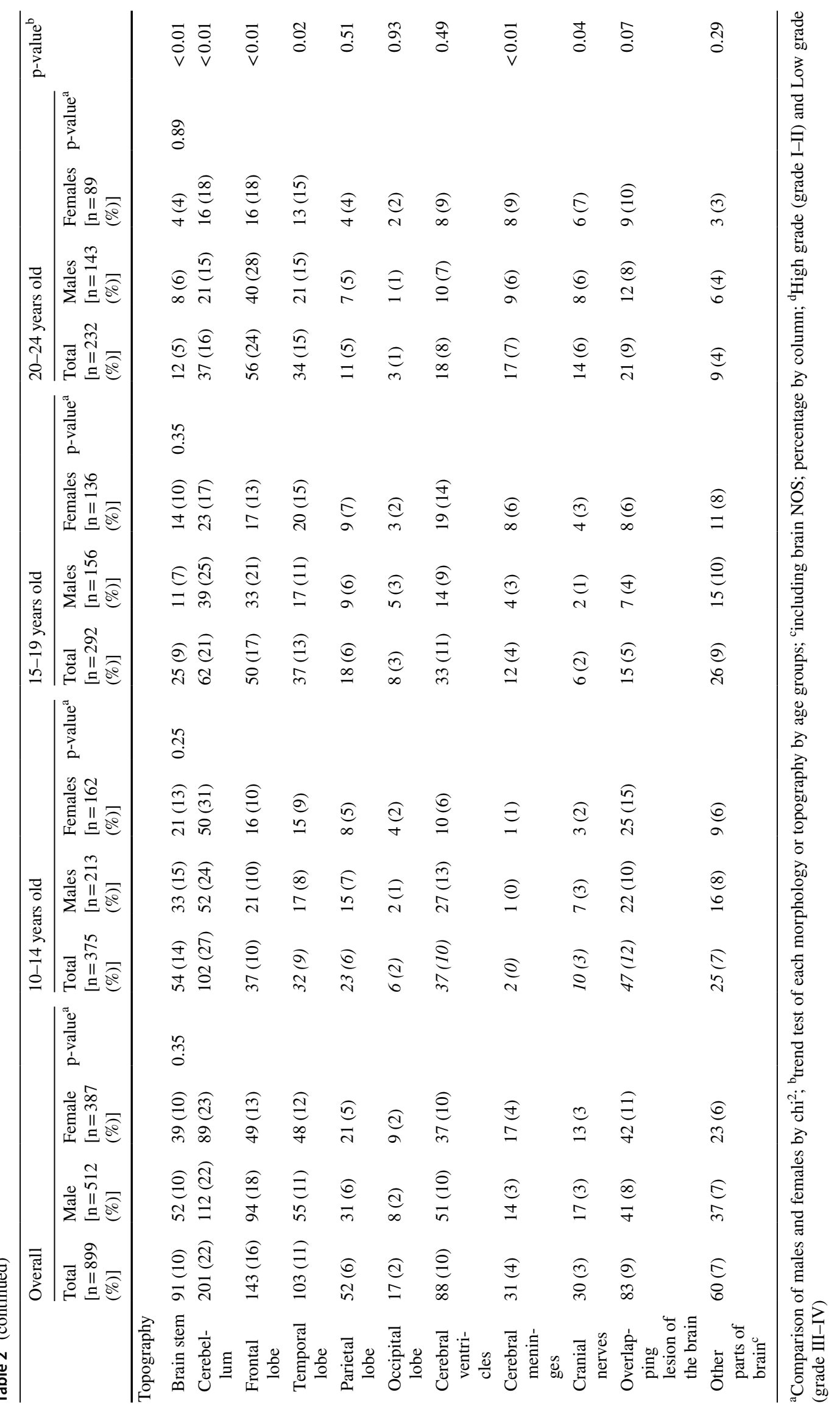




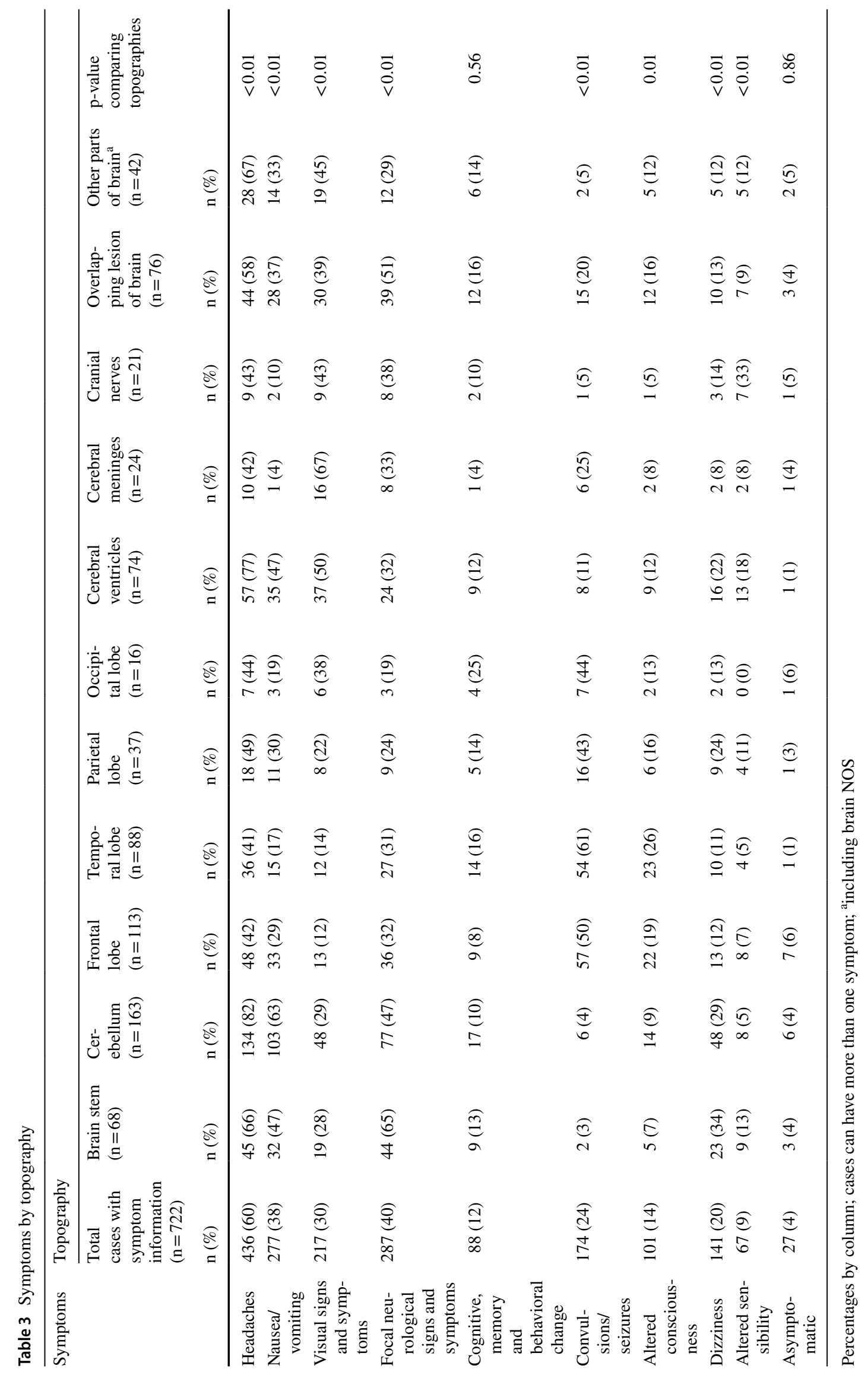


Table 4 Symptoms by grade of tumor

\begin{tabular}{|c|c|c|c|c|c|c|}
\hline \multirow[t]{2}{*}{ Symptoms } & \multicolumn{5}{|l|}{ Grade of tumor (WHO) } & \multirow[t]{2}{*}{ p-value ${ }^{a}$} \\
\hline & $\begin{array}{l}\text { Total cases with } \\
\text { symptom information } \\
(\mathrm{n}=722)\end{array}$ & $\mathrm{I}[\mathrm{n}=308(\%)]$ & II $[\mathrm{n}=144(\%)]$ & III $[\mathrm{n}=113(\%)]$ & $\operatorname{IV}[\mathrm{n}=157(\%)]$ & \\
\hline Headaches & 436 & $181(59)$ & $69(48)$ & $68(60)$ & $118(75)$ & $<0.01$ \\
\hline Nausea/vomiting & 277 & $101(33)$ & $34(24)$ & $47(42)$ & $95(61)$ & 0.11 \\
\hline Visual signs and symptoms & 217 & $93(30)$ & $33(23)$ & $37(33)$ & $54(34)$ & 0.11 \\
\hline $\begin{array}{l}\text { Focal neurological signs and symp- } \\
\text { toms }\end{array}$ & 288 & $115(37)$ & $50(35)$ & $53(47)$ & $70(45)$ & 0.07 \\
\hline $\begin{array}{l}\text { Cognitive, memory and behavioral } \\
\text { change }\end{array}$ & 88 & $37(12)$ & $13(9)$ & $16(14)$ & $22(14)$ & 0.39 \\
\hline Convulsions/seizures & 174 & $75(24)$ & $58(41)$ & $22(20)$ & $19(12)$ & $<0.01$ \\
\hline Altered consciousness & 101 & $48(16)$ & 28 (19) & $8(7)$ & $17(11)$ & 0.02 \\
\hline Dizziness & 141 & $54(18)$ & $16(11)$ & $28(25)$ & $43(29)$ & $<0.01$ \\
\hline Altered sensibility & 67 & $27(9)$ & $16(11)$ & $15(13)$ & $9(6)$ & 0.13 \\
\hline Asymptomatic & 27 & $13(4)$ & $7(5)$ & $2(2)$ & $5(3)$ & 0.63 \\
\hline
\end{tabular}

Percentages by row; ${ }^{\mathrm{a}} \mathrm{p}$-value for comparison of grades; cases can have more than one symptom

observations include headaches with visual signs and symptoms and focal signs and symptoms in embryonal tumors; headaches with visual signs and symptoms and nauseas/ vomiting with dizziness in meningiomas; cognitive changes with convulsions/seizures in 'other non-neuroepithelial tumors'; headaches with visual signs and symptoms, dizziness and focal signs and symptoms and convulsions/seizures with altered consciousness in brainstem tumors; headaches, nausea/vomiting and focal signs and symptoms in cerebellar tumors; cognitive changes and convulsions/seizures in occipital lobe tumors.

\section{Time between symptoms onset and diagnosis}

Time between occurrence of symptoms and final diagnosis was available for $91 \%$ of the cases (Table 5). Most (52\%) were diagnosed within 2 months of first reported symptom, while $12 \%$ were diagnosed over 1 year later; of these, half were diagnosed over 2 years after first symptoms, including 13 cases 5 years or more later.

The median time between the earliest symptom and diagnosis ranged from 0.80 (for visual signs and symptoms and altered consciousness) to 2.7 months (for altered sensitivity) (Supplementary Table A10). Among neuroepithelial tumors, gliomas had a shorter median time (1.10 months and 1.37 months for high- and low-grade tumors respectively) than other tumors (2.57 months). We did not find statistical differences among non-neuroepithelial tumors (Supplementary Table A8).

Regarding topography, the median time ranged from 1.07 to 1.83 months for brainstem, cerebellar, frontal lobe, cerebral ventricles, cerebral meninges, brain NOS and occipital lobe; it was somewhat longer for other tumors: 2-2.2 months for tumors in the temporal and parietal lobes, 3 months for cranial nerves tumors (Supplementary Table A8).

A common sequence of symptoms was observed in most morphologies and topographies: if headache was the first symptom, nausea/vomiting was the most frequent concurrent or following symptom, and focal neurological signs and symptoms the third (Supplementary Table A12).

We also analyzed time from the earliest and latest symptoms to diagnosis (Supplementary Table A10). The earliest symptom was headaches in 59\% of cases overall (median time to diagnosis of 1.27 months), $52 \%$ of gliomas and $73 \%$ of embryonal tumors. Other frequent earliest symptoms were focal and neurological signs and symptoms (27\%) and nausea/vomiting (33\%). Median time was shortest for visual signs and symptoms and altered consciousness ( 0.8 months). The longest median time was observed for most morphologies when altered sensitivity was the earliest symptom. For embryonal tumors it was for convulsions/seizures and for meningioma cognitive, memory and behavioral changes. For the latest symptom (Supplementary Table A10), headache was the most frequently reported (47\%) for all tumors except meningioma and other neuroepithelial tumors. Other frequently registered latest symptoms were nausea/vomiting (33\%) and focal neurological signs and symptoms (32\%). Median time to diagnosis was shortest for cases whose latest symptom was visual sign and symptoms or altered consciousness (0.50 months) and longest for dizziness (1.10 months).

Median time to diagnosis appeared to vary with age and tumor type, but not gender (Supplementary Table A11). For embryonal, cranial nerves, meningiomas and other mesenchymal tumors, time from earliest symptoms to diagnosis decreased with age, while it increased with age for other 


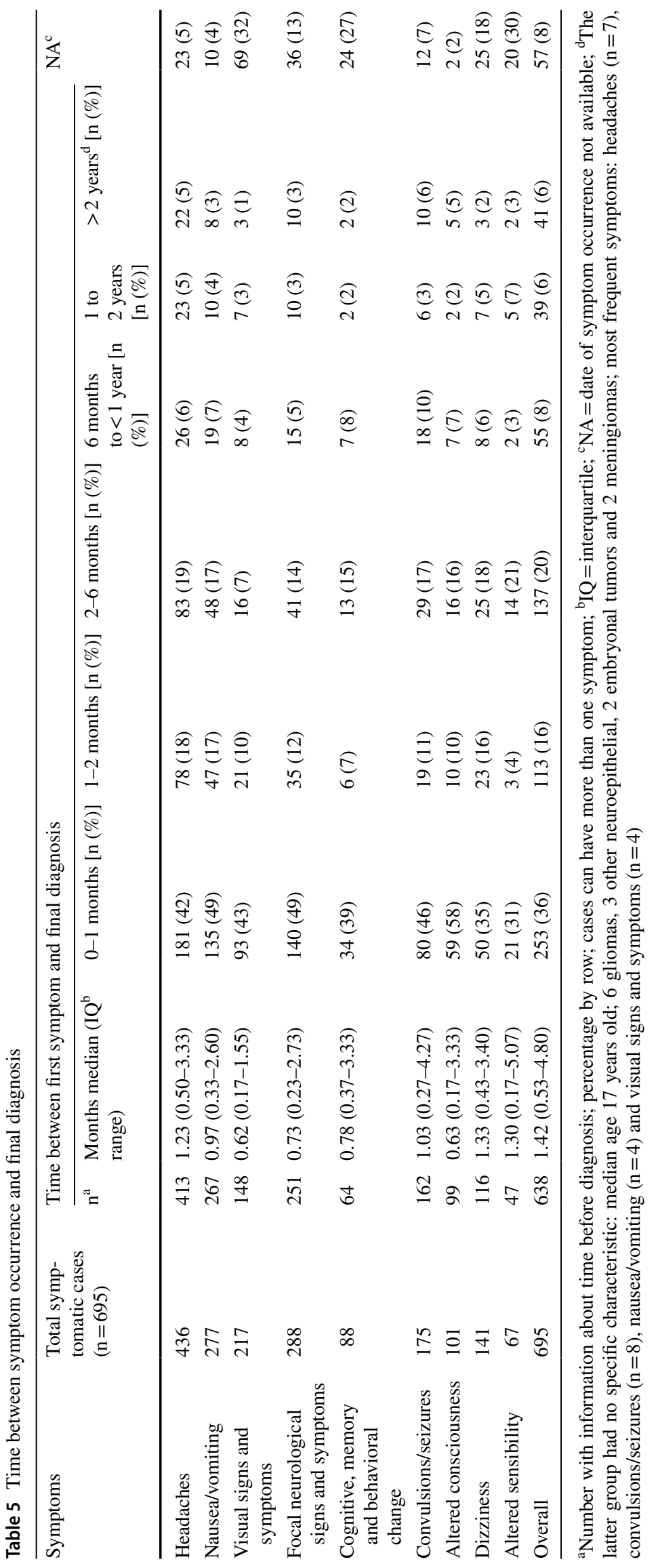


astrocytic tumors. Analyses by topography suggest that time from earliest symptom to diagnosis decreased with age for cerebellum, parietal lobes and overlapping lesion of the brain. The longest time to diagnosis was for brainstem and occipital lobe in the older age group. In terms of time from the latest symptoms, neuronal and mixed neuronal-glial tumors had the longest time to diagnosis.

Analyses of time between the first image that showed a space-occupying lesion and diagnosis showed a median difference of about one week (Supplementary Table A15). For the vast majority of morphologies, cases were diagnosed within 1 month, though the delay was one year or more in $1 \%$ of cases; these showed no specific characteristics: median age 15.5 years; no difference by gender; main symptoms: headaches $(n=5)$, nausea/vomiting $(n=3)$, visual sign and symptoms $(n=6)$, focal neurological signs and symptoms $(n=6)$ and convulsions $(n=3)$.

\section{Discussion}

MOBI-Kids is the largest case-control study of BT in young people conducted so far, allowing investigation of the clinical characteristics of a large number of BT cases from 14 countries.

We found that gliomas were the most frequent tumors type, followed by embryonal tumors, as reported in previous publications $[5,9]$. For topography, the most frequent locations were cerebellum and frontal lobe, as reported in other studies in young adults $[9,10]$. As expected from the literature $[11,12]$, we found a significant decreasing trend with increasing age for embryonal tumors, and an increasing trend with age for meningioma and other non-neuroepithelial tumors. We also found a significant increasing trend with age for frontal and cerebral meninges tumors, but a decreasing trend for brainstem and cerebellum tumors, similar to previous publications [5].

There was a significant difference in the proportion of embryonal tumors by sex (sex ratio-male/female-2.31) which merits further study.

Similar to results of previous meta-analyses [13-15] and other studies $[9,16-20]$, we found a high prevalence of headache, focal neurological signs and symptoms, nausea/vomiting and visual signs and symptoms among our BT cases.

There were significant differences for all symptoms across topographies. Headaches and nausea/vomiting were most frequently reported for posterior fossa tumors as reported by others [15]. Visual signs and symptoms were most frequently reported for cerebral meninges, occipital lobe, and cranial nerves locations, in accordance with the location of the visual cortex and pathways of optical nerve. Interestingly, such symptoms were also frequent in cases with tumors in the cerebral ventricles and infratentorial locations, which may be explained by proximity to visual pathways or cranial nerve nuclei.

The patterns of symptoms, depending on tumor location, grade and morphology, demonstrates a remarkable consistency across studies although the levels of prevalence may differ. We found repeatedly headaches followed by nausea and vomiting as the most frequent symptoms, as reported by others $[9,18]$. Intracranial pressure (ICP) is considered the cause of the joint occurrence of headaches and vomiting (without nausea). In general, variations in symptoms prevalence may be related to differences in ICP due to the growing tumor mass $[10,18,21]$ that can exert pressure not only where the tumor is located but also in other areas.

The widespread use and accessibility of imaging techniques has contributed to earlier diagnosis of CNS tumors but diagnostic latencies are still sometimes of several months $[9,16,18-25]$; there is in fact even evidence that it not been shortened in the past decades [26]. As diagnostic latency increases, the tumor can grow or spread and eventually may lead to increased case fatality and/or long-term neurological and psychological sequelae (depending on the growth characteristics and aggressiveness of the tumor) [27, 28]. It has been suggested that behavioral symptoms (in medulloblastoma) are related with a longer diagnostic delay [29] and it is possible that behavioral changes in teenagers may, at first, be attributed to puberty $[19,30]$. Our results partly confirm this observation, with cognitive, memory and behavioral changes having the longest latency among all symptoms in embryonal tumors, except for rare occurrences of seizures. Though it has been suggested that older children tend to have longer diagnostic delays than younger ones [22], we found no differences between age groups.

The majority of cases were diagnosed within 2 months after first symptom, despite the non-specificity of most signs and symptoms, consistent with earlier findings [17, $23,31-33]$. Longer latencies were found in $12 \%$ of cases, in line with a previous study [21]. There was little difference in symptom prevalence between latency groups, suggesting that the nature of symptoms does not greatly influence diagnostic latency. The median number of symptoms per case was 2 , with some indication of differences by age and gender. Girls may communicate more freely about their symptoms with parents and health-care providers and parents are more aware of symptoms among children than among adolescents and young adults. Because malignant tumors grow faster than benign tumors, they generally lead to increase in ICP earlier, which affects occurrence of symptoms $[16,17,20]$. We found that children with high-grade tumors had a higher number of symptoms and an earlier diagnosis than those with low-grade tumors. Other studies suggested a positive correlation between vomiting and ataxia/gait abnormality and tumor grade, 
with these symptoms being more frequent in high grade tumors [19]. A major challenge in the diagnosis of BTs is that symptoms are often unspecific. Nevertheless, the joint occurrence of multiple symptoms or signs, or their persistent presence should generate awareness of the possibility of BT. Even if an underlying tumor is unlikely, patients and/or care-givers should be encouraged to contact health-care providers again should symptoms persist or progress. According to our results, these symptoms are more frequent for tumors with grade IV but also for grade I, which does not totally agree with earlier findings.

Our study has some limitations. Signs and symptoms were extracted from the clinical documentation of the cases and accuracy of these data depends on the completeness and accurateness of documentation, on the history given by patients and parents/caretakers and the signs detected by the examining physician. However, medical decisions will always be based on patients' histories and findings obtained by examination of the patient rather than by an ideal and full spectrum of facts. We assumed that signs or symptoms not mentioned in the clinical record did not occur. There was no central review of radiology and histopathology although validation studies are under way. Concerning topography, we used up to three sources of evidence: the radiologist's report, the surgery report, and the tumor mapped in a 3-D brain model (XGridMaster) by neuroradiologists. In case of discrepancy, we contacted the regional coordinator who checked, as necessary, with the treating physician. Concerning morphologies, we assigned broad categories that are likely unaffected by slight differences in neuropathologists' categorizations.

Another limitation relates to the exclusion criteria the MOBI-Kids study applied, since tumors located in the midline area were excluded, thereby excluding pituitary tumors that are quite frequent in this age range and preventing us from providing information on these tumors. Further, limiting the age eligibility range to $10-24$ years was a pragmatic solution, and not physiology-based since there is no distinct cut-off at 10 years concerning BT morphology and topography. The upper age of 24 years can arguably be defended by pointing to the well-known transition of BT types around this age. Because we excluded children below 10 years of age, our results are less affected by age-related difficulties to identify and specify symptoms [34]. Another limitation is the omission of information about the time of day symptoms occurred (a shortcoming for headaches that, when occurring in the morning, are considered to be more likely related to BTs).

The main strength is the fact that data were collected using a common protocol in 14 countries around the world. Clinical information was collected shortly after diagnosis and all cases were histologically confirmed. To our knowledge, no other study to date included so many cases in young people, for which data on clinical appearance are largely lacking.

\section{Conclusion}

MOBI-Kids is the largest study of BT in young people conducted so far and it provided the opportunity to study signs, symptoms and other clinical characteristics of BT in young people. Many signs and symptoms were identified, dominated by headaches and nausea/vomiting. We also provided comprehensive information for very rare tumor types to provide input for future reviews.

Though the vast majority of tumors were diagnosed rapidly, within two months from first reported symptoms, $12 \%$ of cases were not diagnosed until at least one year after symptoms. Further studies of the characteristics of tumors with long times to diagnosis may ensure faster access to treatment.

Acknowledgements We acknowledge all research assistants and interviewers in the study centers, participating hospitals, physicians, and especially to all the participants. The Spanish coordinating center would like to thank Alex Albert, Francesc Badia and Ana Espinosa for their invaluable technical and statistical support; Gema Carretero, the Spanish field work coordinator; Eva Ferreras, Irene Gavidia, ${ }^{\mathrm{a}}$ Antonia Muñoz and the other Spanish interviewers for their dedication. We would like to thank specially Dr. José Luis Gil Salu, Dr. Angel Horcajadas, Dr. Ignacio Martín, Dr. Javier Marquez, Dra. Bernarda Márquez, Maria Antonia Muñoz, Carolina Castilla, Fernando Carmona, Dr. Felipe Pareja, Dr. Gómez, Dr. Javier Padillo, Dr. Osama El Rubaidi, Dr. Lozano, Dr. Rainiero Avila, and the rest of clinicians who helped in the provision of information. ISGlobal is a member of the CERCA Programme, Generalitat de Catalunya. We acknowledge support from the Spanish Ministry of Science, Innovation and Universities through the "Centro de Excelencia Severo Ochoa 2019-2023". The Canadian center would like to thank to Fatima Kazoun, Tina Changoor, Agnes Lai, Dr. Michael Vassilyadi, Gail Macartney, Dr. Michael Taccone, Dr. Jean Michaud, Maria Andrews, Brendan Bakos, Ryan Woods, Cathy MacKay, Lisa Stellingwerff, Dr. Brian Toyata, Dr. Thomas J. Zwimpfer, Bonnie Giovannetti, Dr. Juliette Hukin, Colleen Fitzgerald, Jessica Davis, Dr. Eric Bouffet, Mark Bernstein, Dr. Nataliya Zhukova. The Italian centre would like to thank Antonio Argentino, Anna Maria Badiali, Emma Borghetti, Valentina Cacciarini, Laura Davico, Mariangela Farinotti, Laura Fiorini, Francesco Marinelli, Caterina Salce, and all cooperating professionals who made this study possible: Fabio Calbucci, Graziano Guiducci, Mario Lima, Valentina Monti, Andrea Pession, Carmelo Sturiale, Antonella Valentini (Emilia Romagna); Stefania Acerno, Luca Ansaloni, Luciano Beccaria, Francesco Biroli, Ignazio Campanella, Laura Castoldi, Carlo Cesana, Maurizio Cheli, Eugenio Cosenza, Melchiorre Costa, Marta Crespi, Stefano Di Lernia, Giovanni Ferrari, Giuseppe Galli, Valerio Gentilino, Sergio Giombini, Carlo Giussani, Marco Lanata, Francesco Lombardi, Rossella Merli, Pietro Mortini, Gloria Pelizzo, Stefano Peron, Andrea Pietrabissa, Bianca Pollo, Maria Polosa, Paolo M. Rampini, Giorgio Rossi, Vincenzo Rossi, Maurizio Sallusti, Andrea Salmaggi, Carlo L. Solero, Angelo Taborelli, Giuseppe Talamonti, Gian Piero Tonnarelli, Francesco Trotta, Laura Valentini, Marco Zecca, Maria Camilla Zotti (Lombardia); Luca Ambrogio, Andrea Barbanera, Paolo Baroffio, Claudio Bernucci, Elena Codognotto, Franca Fagioli, Luca Grasso, Luciana Lazzarin, Loretta Massone, Antonio Melcarne, Jasmina Micari, Cristina Mocellini, Guido Mondini, Isabella Morra, Chiara Nurisso, Giusy Pace, Elena Riviello, Roberta Rudà, Elisabetta Teruzzi, Elisa Trevisan, Stefano Vallero (Piemonte); Franco Ammannati, Marta Berti, Lorenzo Bordi, Cristina Epifani, Silvia Farina, Lorenzo Genitori, Flavio Giordano, Giuseppe Lippi, Mirko Lombardi, Aurora Mariani, 
Giovanni Marcocci, Antonio Messineo, Giuseppe Olivieri, Iacopo Sardi, Francesco Spina, Andrea Valeri, Patrizia Verdelli (Toscana). The Indian center would like to thank Divya Patel, Nayana Nalawade, Nimisha Mishra and other interviewers for their continuous efforts and dedication, and also to Ankita Manjrekar for her efforts in the Data Management.

Authors' contributions All authors contributed to the study conception and design. Literature search, data collection, data curation, planning paper, formal analysis, methodology, interpretation of data, investigation, writing the first draft of the manuscript, figures and tables: AZ-M. Study design, data collection, formulation of objectives, methodology, investigation, interpretation of data, funding acquisition critical review for important intellectual content and reviewing and editing paper: MK, JA, GC-V, EC. Writing group (study design, data collection, formulation of objectives and methods, interpretation of data, critical review the paper): EP, MKG, MMS-V, SS, SP, RN, GF, H-PH. All others (study design, data collection, funding acquisition and reviewing the paper): MM, AW, RD, AM, TW, FM, BL, SM, JS, RV, MH, NY, DK, PR, CM, TR, EB, NK, AE, AT, HL. All authors commented on previous versions of the manuscript. All authors read and approved the final manuscript.

Funding This work was supported by the MOBI-Kids study and the work in this study was obtained from the European Community's Seventh Framework Programme under Grant Agreements Number 226873 - the MOBI-Kids Project-and 603794-the GERoNiMO project. Additional funds for the coordination of MOBI-Kids were obtained from the Spanish Ministry of Science and Innovation (MINECO), while complementary funds for the conduct of MOBI-Kids in Spain were obtained from the Spanish Health Research Fund (FIS) of the National Institute for Health Carlos III. Italian participation is partially supported by a Ministry of Health grant (RF-2009-1546284). In Canada, funding was provided by a grant from the Canadian Institutes for Health Research under a peer-reviewed university-industry partnership program that involved the Canadian Wireless Telecommunications Association, who provided technical data on wireless telecommunications practices in Canada. The German study centre received additional support by the Federal Office for Radiation Protection (BfS). Japanese participation in MOBI-Kids was supported by the Ministry of Internal Affairs and Communications. New Zealand participation was supported by Health Research Council and Cure Kids. In France, this study received funds from the French National Agency for Sanitary Safety of Food, Environment and Labour (ANSES, contract FSRF2008-3), French National Cancer Institute (INCa), Pfizer Foundation and League against cancer. India received funding from Board of Research in Nuclear Sciences (BRNS). The funding sources had no role in: the study design; the collection, analysis, and interpretation of data; the writing of the report; and the decision to submit the article for publication.

\section{Compliance with ethical standards}

Conflict of interest The authors declare that they have no conflict of interest.

Ethical approval Ethics committee approvals for conducting the study were obtained from all national and regional review boards. An informed consent was provided to all participants.

Open Access This article is licensed under a Creative Commons Attribution 4.0 International License, which permits use, sharing, adaptation, distribution and reproduction in any medium or format, as long as you give appropriate credit to the original author(s) and the source, provide a link to the Creative Commons licence, and indicate if changes were made. The images or other third party material in this article are included in the article's Creative Commons licence, unless indicated otherwise in a credit line to the material. If material is not included in the article's Creative Commons licence and your intended use is not permitted by statutory regulation or exceeds the permitted use, you will need to obtain permission directly from the copyright holder. To view a copy of this licence, visit http://creativecommons.org/licenses/by/4.0/.

\section{References}

1. IARC-Globocan (2012) Globocan 2012. International Agency for Research on Cancer, Lyon, France. https://gco.iarc.fr/. Accessed 23 Dec 2016

2. McKinney PA (2005) Central nervous system tumours in children: epidemiology and risk factors. Bioelectromagn Suppl 7:S60-68. https://doi.org/10.1002/bem.20149

3. GBD 2016 Brain and Other CNS Cancer Collaborators (2019) Global, regional, and national burden of brain and other CNS cancer, 1990-2016: a systematic analysis for the Global Burden of Disease Study 2016. Lancet Neurol 18:376-393. https://doi. org/10.1016/S1474-4422(18)30468-X

4. Emerson JC, Malone KE, Daling JR, Starzyk P (1991) Childhood brain tumor risk in relation to birth characteristics. J Clin Epidemiol 44:1159-1166. https://doi.org/10.1016/0895-4356(91)90148 $-3$

5. McKinney PA (2004) Brain tumours: ***incidence, survival, and aetiology. J Neurol Neurosurg Psychiatry 75(Suppl 2):12-17

6. Azizi AA, Hessler K, Leiss U et al (2017) From symptom to diagnosis-the prediagnostic symptomatic interval of pediatric central nervous system tumors in Austria. Pediatr Neurol 76:27-36. https ://doi.org/10.1016/j.pediatrneurol.2017.08.006

7. Wilne S, Koller K, Collier J et al (2010) The diagnosis of brain tumours in children: a guideline to assist healthcare professionals in the assessment of children who may have a brain tumour. Arch Dis Child 95:534-539. https://doi.org/10.1136/adc.2009.162057

8. Sadetzki S, Langer CE, Bruchim R et al (2014) The MOBI-kids study protocol: challenges in assessing childhood and adolescent exposure to electromagnetic fields from wireless telecommunication technologies and possible association with brain tumor risk. Front Public Health. https://doi.org/10.3389/fpubh.2014.00124

9. Molineus A, Boxberger N, Redlich A, Vorwerk P (2013) Time to diagnosis of brain tumors in children: a single-centre experience. Pediatr Int 55:305-309. https://doi.org/10.1111/ped.12095

10. Prasad KSV, Ravi D, Pallikonda V, Raman BVS (2017) Clinicopathological study of pediatric posterior fossa tumors. J Pediatr Neurosci 12:245-250. https://doi.org/10.4103/jpn.JPN_113_16

11. Johannesen TB, Angell-Andersen E, Tretli S et al (2004) Trends in incidence of brain and central nervous system tumors in Norway, 1970-1999. Neuroepidemiology 23:101-109. https://doi. org/10.1159/000075952

12. Walker D, Bendel A, Stiller C et al (2017) Central Nervous system tumors. In: Bleyer A, Barr R, Ries L, et al. (eds) Cancer in adolescents and young adults. Springer International Publishing, Cham, pp 335-381

13. The Childhood Brain Tumor Consortium (1991) The epidemiology of headache among children with brain tumor. Headache in children with brain tumors. The childhood brain tumor consortium. J Neurooncol 10:31-46

14. Udaka YT, Packer RJ (2018) Pediatric brain tumors. Neurol Clin 36:533-556. https://doi.org/10.1016/j.ncl.2018.04.009

15. Wilne S, Collier J, Kennedy C et al (2007) Presentation of childhood CNS tumours: a systematic review and meta-analysis. Lancet 
Oncol 8:685-695. https://doi.org/10.1016/S1470-2045(07)70207 $-3$

16. Dorner L, Fritsch MJ, Stark AM, Mehdorn HM (2007) Posterior fossa tumors in children: how long does it take to establish the diagnosis? Childs Nerv Syst 23:887-890. https://doi.org/10.1007/ s00381-007-0323-8

17. Fukuoka K, Yanagisawa T, Suzuki T et al (2014) Duration between onset and diagnosis in central nervous system tumors: impact on prognosis and functional outcome: onset and diagnosis in CNS tumors. Pediatr Int 56:829-833. https://doi.org/10.1111/ped.12369

18. Hayashi N, Kidokoro H, Miyajima Y et al (2010) How do the clinical features of brain tumours in childhood progress before diagnosis? Brain Dev 32:636-641. https://doi.org/10.1016/j.brain dev.2009.10.001

19. Reulecke BC, Erker CG, Fiedler BJ et al (2008) Brain tumors in children: initial symptoms and their influence on the time span between symptom onset and diagnosis. J Child Neurol 23:178183. https://doi.org/10.1177/0883073807308692

20. Wilne S, Collier J, Kennedy C et al (2012) Progression from first symptom to diagnosis in childhood brain tumours. Eur J Pediatr 171:87-93. https://doi.org/10.1007/s00431-011-1485-7

21. Mehta V, Chapman A, McNeely PD et al (2002) Latency between symptom onset and diagnosis of pediatric brain tumors: an Eastern Canadian geographic study. Neurosurgery 51:365-372

22. Dang-Tan T, Franco EL (2007) Diagnosis delays in childhood cancer: a review. Cancer 110:703-713. https://doi.org/10.1002/ cncr.22849. (discussion 372-373)

23. Dobrovoljac M, Hengartner H, Boltshauser E, Grotzer MA (2002) Delay in the diagnosis of paediatric brain tumours. Eur J Pediatr 161:663-667. https://doi.org/10.1007/s00431-002-1088-4

24. Klitbo DM, Nielsen R, Illum NO et al (2011) Symptoms and time to diagnosis in children with brain tumours. Dan Med Bull 58:A4285

25. Veneroni L, Mariani L, Lo Vullo S et al (2013) Symptom interval in pediatric patients with solid tumors: adolescents are at greater risk of late diagnosis. Pediatr Blood Cancer 60:605-610. https:// doi.org/10.1002/pbc. 24312
26. Goldman RD, Cheng S, Cochrane DD (2017) Improving diagnosis of pediatric central nervous system tumours: aiming for early detection. CMAJ Can Med Assoc J J Assoc Medicale Can 189:E459-E463. https://doi.org/10.1503/cmaj.160074

27. de Ruiter MA, van Mourik R, Schouten-van Meeteren AYN et al (2013) Neurocognitive consequences of a paediatric brain tumour and its treatment: a meta-analysis. Dev Med Child Neurol 55:408417. https://doi.org/10.1111/dmcn. 12020

28. Reimers TS, Ehrenfels S, Mortensen EL et al (2003) Cognitive deficits in long-term survivors of childhood brain tumors: Identification of predictive factors. Med Pediatr Oncol 40:26-34. https ://doi.org/10.1002/mpo.10211

29. Brasme JF, Chalumeau M, Doz F et al (2012) Interval between onset of symptoms and diagnosis of medulloblastoma in children: distribution and determinants in a population-based study. Eur J Pediatr 171:25-32. https://doi.org/10.1007/s00431-011-1480-z

30. Madhusoodanan S, Ting MB, Farah T, Ugur U (2015) Psychiatric aspects of brain tumors: a review. World J Psychiatry 5:273

31. Preuß M, Preiss S, Syrbe S et al (2015) Signs and symptoms of pediatric brain tumors and diagnostic value of preoperative EEG. Childs Nerv Syst 31:2051-2054. https://doi.org/10.1007/s0038 1-015-2842-Z

32. Ramaswamy V, Remke M, Shih D et al (2014) Duration of the prediagnostic interval in medulloblastoma is subgroup dependent: pre-diagnostic interval and medulloblastoma subgroups. Pediatr Blood Cancer 61:1190-1194. https://doi.org/10.1002/pbc.25002

33. Stocco C, Pilotto C, Passone E et al (2017) Presentation and symptom interval in children with central nervous system tumors. A single-center experience. Childs Nerv Syst 33:2109-2116. https ://doi.org/10.1007/s00381-017-3572-1

34. Jariyakosol S, Peragallo J (2015) The effects of primary brain tumors on vision and quality of life in pediatric patients. Semin Neurol 35:587-598. https://doi.org/10.1055/s-0035-1563571

Publisher's Note Springer Nature remains neutral with regard to jurisdictional claims in published maps and institutional affiliations.

\title{
Affiliations
}

\begin{abstract}
Angela Zumel-Marne ${ }^{1,2,3} \cdot$ Michael Kundi $^{4} \cdot$ Gemma Castaño-Vinyals $^{1,2,3,5} \cdot$ Juan Alguacil $^{3,6} \cdot$ Eleni Th Petridou $^{7,8}$. Marios K. Georgakis ${ }^{7}$. Maria Morales-Suárez-Varela ${ }^{3,9} \cdot$ Siegal Sadetzki $^{10,11} \cdot$ Sara Piro $^{12} \cdot$ Rajini Nagrani $^{13,14}$. Graziella Filippini $^{15} \cdot$ Hans-Peter Hutter ${ }^{4} \cdot$ Rajesh Dikshit $^{13} \cdot$ Adelheid Woehrer $^{16} \cdot$ Milena Maule $^{17}$. Tobias Weinmann $^{18}$. Daniel Krewski ${ }^{19}$. Andrea 't Mannetje ${ }^{20}$. Franco Momoli $^{19}$. Brigitte Lacour ${ }^{21,22}$. Stefano Mattioli ${ }^{23}$. John J. Spinelli ${ }^{24,25} \cdot$ Paul Ritvo $^{26}$. Thomas Remen ${ }^{21}$. Noriko Kojimahara ${ }^{27}$. Amanda Eng ${ }^{20}$. Angela Thurston ${ }^{20} \cdot{\text { Hyungryul } \mathrm{Lim}^{28} \cdot \mathrm{Mina} \mathrm{Ha}^{28} \cdot \mathrm{Naohito} \mathrm{Yamaguchi}^{27} \text {. Charmaine Mohipp }}^{29,30} \cdot$ Evdoxia Bouka $^{7}$. Chelsea Eastman ${ }^{1,2,3} \cdot$ Roel Vermeulen $^{31} \cdot$ Hans Kromhout $^{31} \cdot$ Elisabeth Cardis ${ }^{1,2,3}$
\end{abstract}

1 ISGlobal, Barcelona, Spain

2 Universitat Pompeu Fabra (UPF), Barcelona, Spain

3 Ciber Epidemiología y Salud Pública (CIBERESP), Madrid, Spain

4 Center for Public Health, Department of Environmental Health, Medical University Vienna, Vienna, Austria

5 IMIM (Hospital del Mar Medical Research Institute), Barcelona, Spain
6 Environmental Epidemiology and Neuroscience Laboratory, RENSMA, Huelva University, Av. Andalucía s/n, E-21071 Huelva, Spain

7 Department of Hygiene, Epidemiology and Medical Statistics, Medical School, National and Kapodistrian University of Athens, Athens, Greece

8 Clinical Epidemiology Unit Karolinska Institutet, Stockholm, Sweden 
9 Área de Medicina Preventiva y Salud Pública, Universitat de Valencia, Valencia, Spain

10 Public Health Services, Ministry of Health, Tel Aviv, Israel

11 Sackler Faculty of Medicine, Tel Aviv University, Tel Aviv, Israel

12 Environmental and Occupational Epidemiology Branch, Cancer Risk Factors and Lifestyle Epidemiology Unit, Institute for Cancer Research Prevention and Clinical Network-ISPRO, Florence, Italy

13 Centre for Cancer Epidemiology, Tata Memorial Centre, Mumbai, India

14 Leibniz Institute for Prevention Research and Epidemiology - BIPS, Bremen, Germany

15 Scientific Director's Office, Fondazione IRCCS Istituto Neurologico Carlo Besta, Milan, Italy

16 Institute of Neurology, Medical University Vienna, Vienna, Austria

17 Unit of Cancer Epidemiology, Department of Medical Sciences, University of Turin, Turin, Italy

18 Institute and Clinic for Occupational, Social and Environmental Medicine, University Hospital, LMU Munich, Munich, Germany

19 School of Epidemiology and Public Health, University of Ottawa, Ottawa, Canada
20 Centre for Public Health Research, Massey University, Wellington, New Zealand

21 French National Registry of Childhood Solid Tumors, CHU, Nancy, France

22 Inserm, Center of Research in Epidemiology and StatisticS (CRESS), Epidemiology of Childhood and Adolescent Cancers Team (EPICEA), Paris University, Paris, France

23 Department of Medical and Surgical Sciences (DIMEC), University of Bologna, Bologna, Italy

24 Population Oncology, BC Cancer, Vancouver, Canada

25 School of Population and Public Health, University of British Columbia, Vancouver, Canada

26 School of Kinesiology and Health Science and Department of Psychology, York University, Toronto, Canada

27 Department of Public Health, Tokyo Women's Medical University, Tokyo, Japan

28 Department of Preventive Medicine, Dankook University College of Medicine, Cheonan, South Korea

29 Children's Hospital of Eastern Ontario, Ottawa, ON, Canada

30 University of Ottawa, Ottawa, ON, Canada

31 Division Environmental Epidemiology, Institute for Risk Assessment Sciences, Utrecht University, Utrecht, The Netherlands 